\title{
Development and validation of the blanket First Wall mock-up model in RELAP5-3D
}

\author{
Valentino Di Marcello*, Bradut-Eugen Ghidersa, Xue Zhou Jin, Ali Abou-Sena, Robert Stieglitz \\ Karlsruhe Institute of Technology (KIT), Karlsruhe, Germany \\ *Corresponding author: valentino.marcello@kit.edu
}

The Helium Cooled Pebble Bed (HCPB) blanket concept is one of the DEMO (Demonstration Power Plant) blanket concepts running for the final DEMO design selection. For the development of the equatorial outboard blanket module of the HCPB blanket concept, the qualification and testing of mock-ups are foreseen by means of different experiments is mandatory to qualify the thermomechanical and thermal-hydraulics robustness of the design. Therefore, a functional test mock-up of the first wall (FWMU) is planned to be integrated in the HELOKA facility (helium loop Karlsruhe) at KIT. Aside from nominal operation conditions, additionally coolant flow control cycling and typical safety relevant transients are to be studied to provide a database for design tool validation.

This work is aimed to derive a prototypical experimental set-up simulating a LOFA (Loss Of Flow Accident) in a first wall mock-up (FWMU) plate consisting of ten cooling channels, which has been developed in a previous study. In order to identify adequate test parameters allowing to extrapolate the experiments to a DEMO scale and simultaneously matching the experimental constraints, a FWMU model has been developed by means of the system code RELAP5-3D version 4.3.4. Therefore, in a first step, the results of the RELAP model are compared with a CFX analyses of a single channel and two channels representation for both steady-state and transient conditions to verify the system code. In this context an excellent agreement has been obtained. Subsequently, the mock-up pressure loss characteristics have been experimentally determined, requiring a calibration of the 10 channels RELAP model. This model is integrated into HELOKA loop model to simulate different operational scenarios, but also to optimize the piping layout and instrumentation set-up.

Keywords: DEMO, HCPB, LOFA, RELAP.

\begin{tabular}{l|l}
\hline Topic Category & Safety Issues and Waste Management \\
\hline Presentation Preference & $\square$ Oral Presentation $囚$ Poster Presentation \\
\hline
\end{tabular}

This work has been carried out within the framework of the EUROfusion Consortium and has received funding from the Euratom research and training programme 2014- 2018 under grant agreement No 633053. The views and opinions expressed herein do not necessarily reflect those of the European Commission. 